\section{All in the mind}

John C. Marshall

Cognitive Neuropsychology: A Clinical Introductlon. By Rosaleen A. McCarthy and Elizabeth K. Warrington. Academic: 1990. Pp.428. Hbk £41, \$69; pbk £21, $\$ 34.95$.

Neuropsychological Impairments of Short-Term Memory. Edited by Guiseppe Vallar and Tim Shallice. Cambridge University Press: 1990. Pp. 524. $£ 40, \$ 64.50$.

A SIGNIFICANT part of current research in the 'decade of the brain' is characterized by an adverse ratio of hype to honest toil. All too often, the vogue for neural computation has brought forth neurophysiologically implausible models where the relationship between network performance and hard behavioural data ranges between false and mystical. There is excitement aplenty but rather little to show for the increased arousal levels. By contrast, much of 'normal' psychology is all too normal; yet another methodological twist to an experimental paradigm that produces small effects of limited relevance to anything other than the investigator's publication rate.

It is with some relief, then, that I turn to two new books on cognitive neuropsychology. Although I cannot claim to be an unbiased observer, it is nonetheless fair to remark that this discipline is intrinsically forced to confront a very practical reality: the existence of people with acquired brain damage whose cognitive impairment has seriously reduced their capacity for productive work and meaningful leisure. The standard neurological examination, combined with laboratory investigations and neuroimaging techniques will (usually) reveal the proximal medical cause of the patient's condition. But it remains for the neuropsychologist to elucidate the patient's functional diagnosis, prognosis, and rehabilitation potential against a background of normal cognitive processing.

\section{NEW JOURNALS ISSUE}

This year, Nature's annual new journals review supplement will appear in the issue of 3 October. Publishers and learned societies are invited to submit journals for review, taking note of the following criteria:

- Journals that first appeared after June 1989 and issued at least four separate numbers by the end of April 1991 will be considered. The deadline for submission is end of May Journals covering any aspect of science are eligible, but those dealing with clinical medicine, engineering and pure mathematics are excluded, as are publications of abstracts Frequency of publication must be at least three times a year - The main language used must be English.

When submitting journals for review, please send at least four different issues (the first, the most recent and any two others).
Cognitive Neuropsychology: A Clinical Introduction by $\mathrm{R}$. McCarthy and $\mathrm{E}$. Warrington is, quite simply, the clearest, most comprehensive, and up-to-date account of the discipline in print. The volume's coverage is exemplary for those areas where a substantial database exists: object and face recognition, spatial perception, voluntary action, perception, comprehension and production of language, reading and writing, arithmetic calculation, memory, and problem solving. As indicated, the book is organized by functional categories rather than disease entities; different aetiologies often provoke very similar behavioural impairments.

For each domain, McCarthy and Warrington first outline the basic empirical characteristics of how performance can break down within a particular subsystem. Much of the expository burden rests upon the detailed analysis of individual cases who show an especially 'pure' or extreme disorder (or small set of related symptoms). Group studies (or, better, series of well-investigated cases) are then used to reveal the (gross) loci of anatomical damage that has caused the problem. Each chapter concludes with a (brief) theoretical section in which deficits are related to a functional architecture of normal cognition; this architecture is usually expressed as a flow chart that diagrams how "different (processing) components are brought together to perform a specific task." Finally, functional pathology is interpreted as impairment to individual computational centres and the transfer of information between them.

Given the task that McCarthy and Warrington set themselves, it is difficult to imagine that it could have been better done. The book is, however, an introduction, as the title states. A few areas are ignored (disorders of time estimation and of music, for example). Cognitive aspects of personality and thought disorder after focal lesion receive short shrift; what little is included on these topics is hidden away behind attentional or problem-solving impairments. Prognosis and remediation are likewise not covered. There is good reason for these omissions: namely, a relative paucity of reliable and insightful studies. But I would have liked to see more attention paid to the evolution of cognitive deficits. Change in performance over time (manifest either as spontaneous recovery or as progressive deterioration) can provide dynamic constraints on theory that are difficult (or impossible) to obtain from any cross-sectional investigation.

Space limitations must also dictate selectivity in a book of this breadth; a point forcibly brought home by the fact that Neuropsychological Impairments of Short-Term Memory, edited by Giuseppe Vallar and Tim Shallice, can devote 500 or so pages to one neuropsychological symptom complex without padding or repetition. But repetition is the topic. Patients with left posterior parietal damage have severe difficulties in recalling lists (random sequences of words, letters, or numbers, for example) immediately after hearing them. The phenomenon first assumed major theoretical significance when, in the 1960s, Warrington and Shallice documented a surprising dissociation: some patients with dramatically reduced auditoryverbal spans (tested by immediate repetition after one presentation) showed normal learning and long-term memory functions with multiple stimulus presentations.

This finding casts doubt upon what was then the standard account of short-term memory; that it was a necessary 'holding buffer', essential for the transfer of information into long-term storage. The controversy about the relationship between short- and long-term memory has rumbled along ever since, supplemented by an additional question: if the classical account is wrong, then what on earth is the natural function of a short-term, limited-capacity buffer? It is unlikely that the brain should contain a mechanism whose sole purpose is to repeat back telephone numbers once heard.

Vallar and Shallice have assembled a set of outstanding researchers who contribute chapters on just about every aspect of shortterm memory and its neural correlates. In addition to patients with disproportionate difficulty on span tasks after focal lesion, the volume discusses the normal development of immediate memory in children, and the course of its deterioration in healthy older people. Much of the book contains new data and critical discussion of the most plausible hypothesis about the functional role of a short-term buffer: the buffer preserves a low-level phonological representation of sentences just in case the first pass interpretation is wrong (for example, "The teacher always taught by the new method passed the test"). Yet it remains extremely odd that some patients with very restricted spans perform well on tasks where one feels intuitively that a short-term verbatim record must have been preserved.

Such counterintuitive findings are, of course, one important reason why neuropsychological inquiry has played, and continues to play, such a dominant role in the development of cognitive psychology as a serious science. The extreme dissociations in performance seen after acquired brain damage are not random fracture lines. Rather, they reveal an underlying modularity of neuronal mechanisms that is often obscured by an apparent interaction of components in the brain when running smoothly. The painstaking experimental analyses reported in Neuropsychological Impairments of ShortTerm Memory and in Cognitive Neuropsychology: A Clinical Introduction provide theoretical constraints that must be met by formal models of neural computation.

John C. Marshall is at the Neuropsychology Unit, University Department of Clinical Neurology, The Radcliffe Infirmary, Oxford OX2 $6 H E$, UK. 